\title{
Estimativa do Peso Fetal: Comparação Entre um Método Clinico e a Ultra-Sonografia
}

\author{
Estimation of Fetal Weight: Comparison Between a \\ Clinical Method and Ultrasonography
}

Alexandre Faisal Cury, Sidney A. L. Garcia

\section{RESUMO}

Objetivo: avaliar a validade da estimativa do peso fetal por método baseado na altura uterina - regra de Johnson.

Métodos: foram estudadas 101 gestantes e seus recém-nascidos (RN), estimando-se o peso fetal pela utilização da regra de Johnson adaptada, que consiste em aplicação clínica de modelo matemático para cálculo do peso fetal baseado na altura uterina e na altura da apresentação fetal. O peso estimado foi obtido no dia do parto e foi comparado com o peso observado ao nascer, que constituiu o controle da análise da validade do método empregado. Na mesma data foi realizada ultra-sonografia obstétrica (US) detalhada, que inclui cálculo do peso fetal pela aplicação das tabelas de Sheppard, e este peso, estimado pela US, foi comparado ao peso observado ao nascer.

Resultados: os resultados destas comparações mostraram que a estimativa clínica empregada nesta casuistica tem valor semelhante à US para avaliação do peso ao nascer: a margem de acerto do método clínico com variações de 5\%, 10\% e 15\% entre peso estimado e peso observado foi de 55,3\%, 73\% e 86,7\% respectivamente, e, para o US, de 60,7\%, 75,4\% e $91,1 \%$, respectivamente.

Conclusões: quando comparados, estes valores não se mostraram diferentes do ponto de vista estatístico, permitindo concluir-se que a avaliação clínica mostra acurácia semelhante à da US para o cálculo do peso ao nascer.

PALAVRAS-CHAVE: Peso fetal. Ultra-sonografia. Gestação normal.

\section{Introdução}

O peso ao nascer constitui importante variável de risco para mortalidade e morbidade neonatal, sendo clássica a associação entre prematuridade, baixo peso e óbito neonatal,

Maternidade Dr. Cury - Osasco - São Paulo Correspondência:

Alexandre F. Cury

R. Dr Mário Ferraz, 135 / 42 - Jardim Europa

01453-010 - São Paulo - SP particularmente em decorrência da síndrome de desconforto respiratório.

Tal assertiva torna evidente que o cálculo do peso fetal constitui dado da maior importância na propedêutica obstétrica, dado este que, freqüentemente, é fator determinante na decisão clínica de manter ou interromper gestações de risco.

O método atual mais acurado de estimativa do peso fetal é o que utiliza a ultra-sonografia (US), técnica que, necessariamente, depende de equipamento e recursos humanos especializados. No entanto, mesmo com condições técnicas 
adequadas, a estimativa do peso fetal pela US é menos acurada nos casos de feto de peso muito baixo e nos macrossômicos ${ }^{1}$.

A estimativa do peso fetal com base no exame clínico constitui prática pouco difundida e ensinada em nosso meio, embora esta metodologia possa ser de grande valia para a equipe médica nas localidades onde não é disponivel a US. A utilização de métodos clínicos de fácil execução, baixo custo e que possuam propriedades diagnósticas operacionais é desejável, particularmente para a realidade de nosso país.

A literatura não mostra consenso quanto à validade de diferentes métodos clínicos com tal propósito. Assim, Loeffler ${ }^{11}$, utilizando-se apenas da palpação uterina, obteve valores preditivos considerados razoáveis na estimativa do peso fetal. Resultados melhores foram obtidos por autores que utilizaram o produto da multiplicação entre a altura uterina e a circunferência abdominal ${ }^{9,16}$. Também Calvert et al. ${ }^{5}$, Azziz et al. ${ }^{2}$ e Johnson et al. ${ }^{10}$ basearam suas estimativas na mensuração da altura uterina e obtiveram resultados satisfatórios. Belizan ${ }^{4}$ demonstraram validade desta metodologia de avaliação de peso fetal para rastrear retardo de crescimento intra-uterino (RCIU).

Outros autores, como Beazley e Underhill ${ }^{3}$, consideram que a ampla variação da altura uterina mensurada através do abdome materno limita o uso deste parâmetro com tal propósito. Igualmente, Rosemberg et al. ${ }^{14}$ desaprovam o uso desta metodologia no rastreamento clínico do RCIU.

O propósito deste trabalho é avaliar a validade da estimativa do peso fetal por método baseado na altura uterina - regra de Johnson - e comparar esta estimativa com aquela realizada mediante uso da US.

\section{Pacientes e Métodos}

Foram incluídas 101 pacientes admitidas para assistência ao parto na Maternidade Dr. Cury, Osasco, São Paulo, no período de dezembro de 1992 a junho de 1993. Todas as pacientes haviam realizado acompanhamento pré-natal na instituição, tendo a idade gestacional corretamente estabelecida por meio de US até a vigésima semana e/ou data conhecida da última menstruação.

$\mathrm{Na}$ ocasião da admissão para assistência ao parto, todas foram avaliadas clinicamente pelo mesmo obstetra, sendo medida a altura uterina com fita métrica justaposta sobre o abdome materno, com o zero apoiado junto ao bordo superior da sinfise púbica, por mão fixa, e seu nivel superior no fundo uterino, limitado pela borda cubital da mão livre.

Foi realizado toque vaginal bidigital em que, além da avaliação usual, procurou-se detalhar a altura da apresentação. Considerou-se a cabeça insinuada (quando o maior diâmetro da apresentação havia transposto a área do estreito superior), cabeça fixa (quando não se conseguiu mobilizar a cabeça) e cabeça alta e móvel (quando a cabeça não havia tomado contato com o estreito superior) ${ }^{13}$.

O cálculo clínico do peso fetal teve como base a altura uterina e a altura da apresentação, aplicando-se a fórmula baseada na regra de Johnson ${ }^{10}$ :

Peso fetal $=155 \times(\mathrm{AU}-\mathrm{K})$

Onde $\mathrm{AU}=$ altura uterina encontrada

e $\mathrm{K}=11$ (cabeça insinuada), 12 (cabeça fixa) ou 13 (cabeça alta e móvel)

$\mathrm{Na}$ admissão, as pacientes foram submetidas à US, sempre pelo mesmo profissional, que, além da avaliação rotineira do exame, calculava o peso fetal utilizando-se de tabelas de Sheppard et al. ${ }^{15}$ para tal finalidade, que inferem este dado associando a circunferência abdominal e o diâmetro biparietal.

Foram excluídos os casos nos quais o fenômeno da moldagem do polo cefálico impedia adequada mensuração, as amniorrexes prematuras, as apresentações não-cefálicas e as anormalidades no volume de líquido amniótico (oligo e polidrâmnio), tendo sido adotados para este fim os critérios propostos por Chamberlain et al. ${ }^{6,7}$. Para as pacientes com peso superior a $90 \mathrm{~kg}$ foi subtraída uma unidade (1) do valor encontrado para a altura uterina, conforme a regra de Johnson.

Os recém-nascidos (RN) foram pesados em balanças digitais aferidas e tiveram sua idade gestacional estabelecida pelo método de Capurro. Este peso constituiu o padrão para o estudo das variações encontradas entre os pesos estimados pelo método clínico utilizado e a US.

Foi pesquisada a dimensão da variação entre pesos estimados e pesos reais encontrados, pelo estudo da razão entre peso estimado/peso encontrado, tanto para o método clínico quanto para a US e, posteriormente, comparou-se a acurácia de ambos os métodos.

Foram utilizadas tabelas de contingência tipo $2 \times 2$ e teste do $\chi^{2}$ para comparação de resultados obtidos com o método clínico e ultra-sonográfico de avaliação do peso fetal, com variação de 5, 10 e $15 \%$ entre pesos estimados e encontrados. 


\section{Resultados}

A idade das pacientes variou de 17 a 39 anos, com média de 25,7. A idade gestacional variou de 34 a 41 semanas (Figura 1). A moda encontra-se entre 38 e 41 semanas $(89,2 \%)$. Tais freqüências são compativeis com a população assistida na Instituição, onde a prematuridade é encontrada em aproximadamente $10 \%$ dos casos.

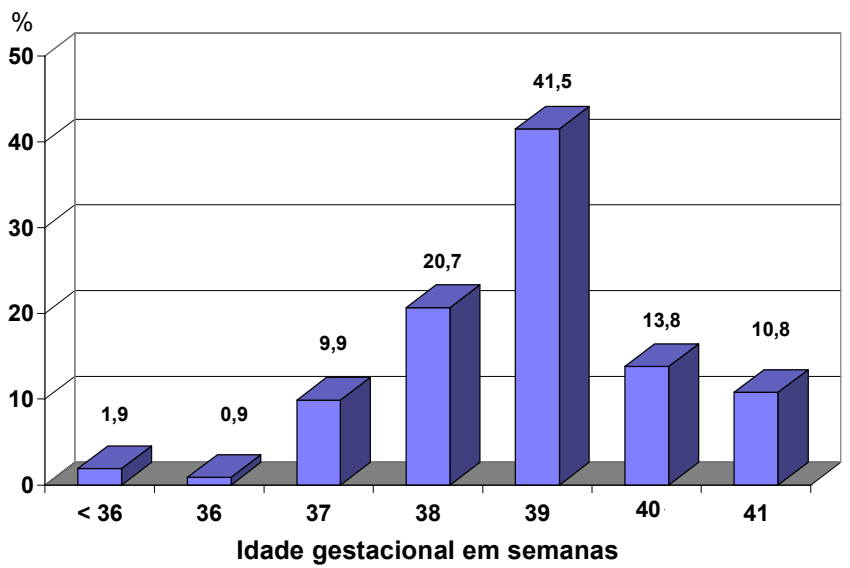

Figura 1 - Distribuição da idade gestacional em semanas.

A distribuição do peso dos RN encontra-se na Figura 2, a maioria dos casos com peso entre 2.500 e 3.999 g $(91,9 \%)$. A relação entre o peso estimado pela regra aplicada e o peso real encontrado ao nascer - PE/PR - encontra-se demonstrada na Figura 3. Este gráfico mostra, por meio de índices, as variações para mais e para menos entre o peso calculado clinicamente e aquele, real, constatado ao nascimento, sendo que a relação igual a 1 mostra acerto de $100 \%$; as abaixo da unidade peso subestimado pela regra, e as acima da unidade pesos superestimados pela regra. A figura ainda demonstra em que niveis as variações foram incorretas, assim valores de 0,90 significam subestimativa de $10 \%$ em relação ao peso real e variações de 1,10 significam superestimativa de $10 \%$. Nota-se, pela distribuição gráfica, que a maioria dos casos (73\%) encontra-se concentrada na faixa entre a unidade e variações até $10 \%$ para mais ou para menos.

Para fins de comparação, foi estimado o peso fetal por US, aplicando-se as tabelas de Sheppard et al. ${ }^{15}$ para cálculo do peso fetal, e procedeu-se à análise dos dados conforme a mesma metodologia.

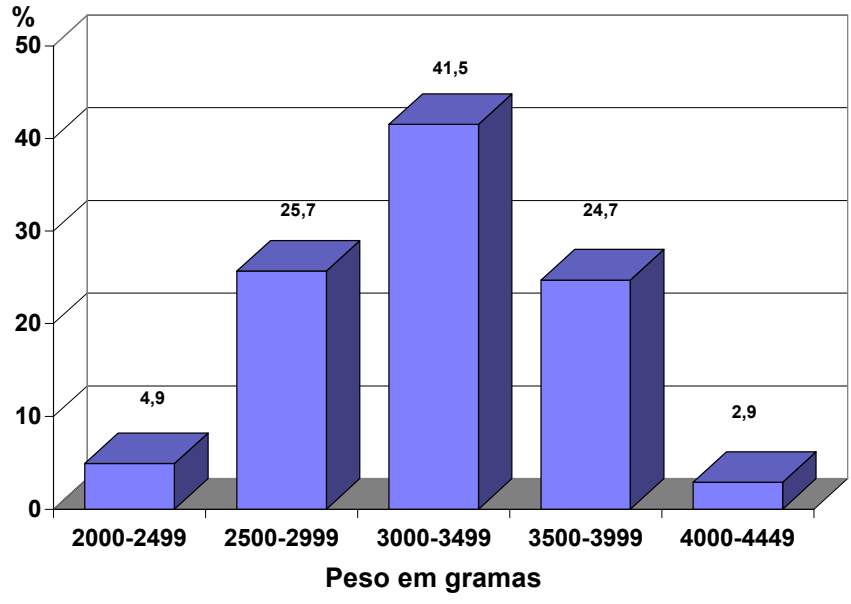

Figura 2 - Distribuição de peso ao nascer em gramas.

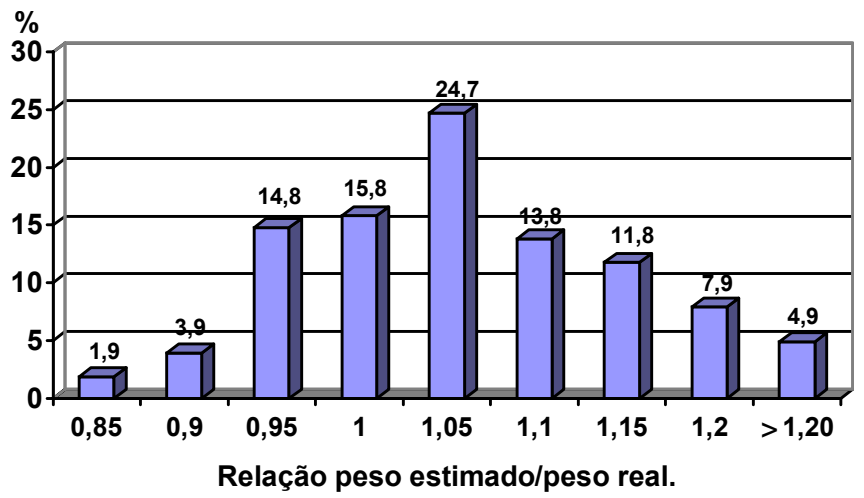

Figura 3 - Relação peso estimado / peso real. Avaliação por método clínico.

Os resultados do cálculo do peso fetal por meio da US comparados com o peso real ao nascer encontram-se na Figura 4 e obedecem à mesma interpretação aplicada ao método anterior. Também foram obtidos valores subestimados e superestimados com uso do US, caracterizados pelas variações acima e abaixo da unidade.

Para efeito de pesquisa da validade do método clínico aplicado, compararam-se os dados obtidos das variações até $5 \%, 10 \%$ e $15 \%$ entre as estimativas de peso realizadas pelo método clínico e pelo US, em relação ao peso real (Figura 5).

As freqüências observadas de variação entre peso estimado e peso encontrado para ambos os métodos (US e regra clínica) são bastante próximas, e o estudo estatístico realizado pelo teste do $\chi^{2}$ não mostra diferença significativa entre os dois métodos, para variações de peso encontrados e estimados de $5 \%(p=0,3917), 10 \%(p=0,6270)$ e $15 \%(\mathrm{p}=0,5063)$. 


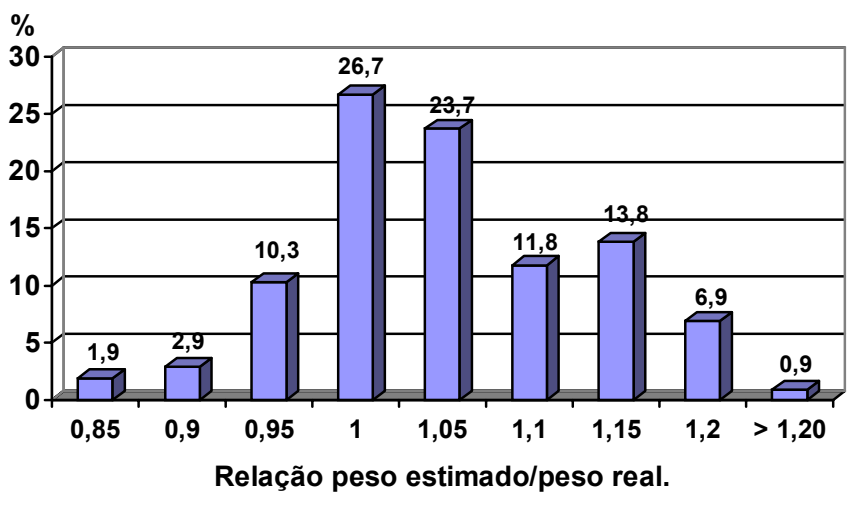

Figura 4 - Relação peso estimado / peso real. Avaliação por ultra-som.

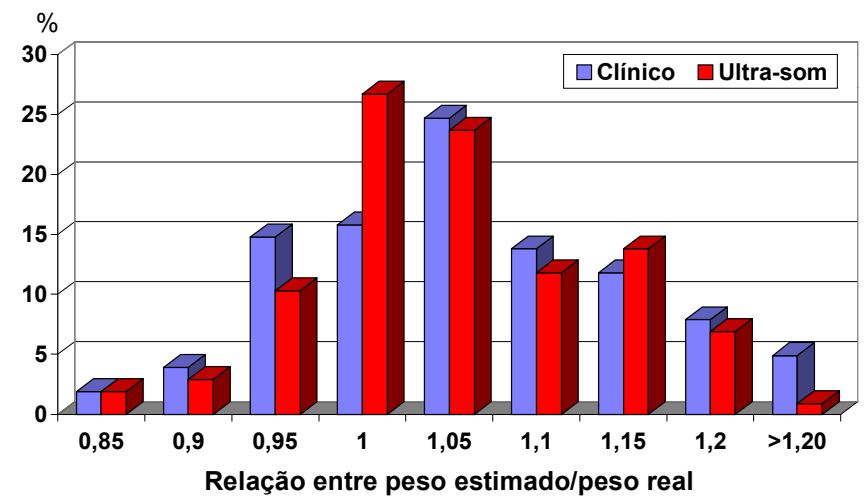

Figura 5 - Relação entre peso estimado e peso real: comparação da acurácia do método clínico com o ultra-som.

\section{Discussão}

O cálculo do peso fetal constitui fator de enorme importância para o embasamento de condutas resolutivas nas gestações de risco, por tratar-se de parâmetro que se relaciona diretamente com morbimortalidade neonatal. $\mathrm{O}$ baixo peso ao nascer é o maior fator de risco em associação ao óbito neonatal.

Torna-se atraente a possibilidade de avaliação do peso fetal por meio de metodologia clínica de baixo nivel de complexidade, aplicável a qualquer nivel assistencial, prescindindo de equipamentos sofisticados e de recursos humanos altamente qualificados e especializados e utilizando parâmetros simples e objetivos, como a medida da altura uterina e a altura da apresentação cefálica fetal.
A aplicação da regra de Johnson, para cálculo do peso fetal mostrou boa correlação com o peso encontrado ao nascer, resultando que, com variações de até $10 \%$ para mais ou para menos, $73 \%$ dos casos tiveram seu peso previsto. Utilizando-se da mesma metodologia, o cálculo do peso fetal realizado pelo US mostrou margem de acerto com variação de até $10 \%$, em $75,4 \%$, valores estes muito próximos daqueles encontrados com a avaliação clínica.

Esta semelhança de acurácia dos métodos pode ser notada na Figura 5, onde se nota claramente a proximidade dos valores encontrados em ambas as formas de avaliação do peso fetal. O fato de as pacientes estarem em trabalho de parto não interferiu na estimativa clínica ou ultrasonográfica do peso fetal. Na literatura existem trabalhos comparando a acurácia das duas metodologias em parturientes. Chauan et al. ${ }^{8}$ compararam a estimativa do peso fetal feita pela própria gestante com as estimativas clínica e ultrasonográfica, em 106 multíparas parturientes. Adotando o limite de até $10 \%$ de variação do peso real, obteve para estimativa materna, clinica e ultrasonográfica acertos de, respectivamente, $69,8 \%$, $66,1 \%$ e $42,4 \%$ As diferenças não-significativas atestam, segundo os autores, a validade do método clínico para cálculo do peso fetal.

Por outro lado, Patterson ${ }^{12}$ estimou o peso fetal por método clínico e duas análises ultrasonográficas em 62 parturientes. As estimativas ultra-sonográficas foram mais acuradas que o método clínico, exceto nos casos nos quais a dilatação cervical era superior a $3 \mathrm{~cm}$. O autor sugere que a estimativa do peso fetal pelo método clínico seja interpretada criticamente, nas diversas situações clínicas do caso.

A avaliação do peso fetal pela regra de Johnson tem aplicação clínica. $\mathrm{Na}$ casuística estudada, a margem de acerto do método, com variações de até $5 \%, 10 \%$ e $15 \%$ entre peso estimado e peso real encontrado ao nascer, foi de $55,3 \%, 73 \%$ e $86,7 \%$, respectivamente. A margem de acerto com o método ultra-sonográfico com variações de ate $5 \%, 10 \%$ e $15 \%$ foram $60,7 \%$, $75,4 \%$ e $91,1 \%$, respectivamente. Sendo a ultrasonografia considerada no momento o método mais preciso para o cálculo do peso fetal, os resultados obtidos com as duas formas de avaliação demonstram a acurácia e operacionalidade clínica da regra de Johnson.

No entanto, novos estudos em gestações de fetos com RCIU e macrossomia, bem como nas gestantes obesas são necessários para confirmar os resultados promissores aqui encontrados com a regra de Johnson. 


\section{SUMMARY}

Purpose: to assess the validity of fetal weight estimation by a method based on uterine height-Johnson's rule.

Methods: one hundred and one pregnant women and their newborn children were studied. The fetal weight was estimated using an adaptation of Johnson's rule, which consists of the clinical application of a mathematical model to calculate the fetal weight based on the uterine height and the height of fetal presentation. The estimated weight was obtained on the day of delivery and was compared to the weight observed after birth. This, in turn, was the control of the analysis of validity of the method used. On the same date, a detailed obstetrical ultrasonography (US) was conducted which included the fetal weight, calculated by the use of Sheppard's tables. This weight, estimated by US, was compared to the birth weight.

Results: the results have proven that the clinical estimate used in this study has a similar value to that of the US calculation of birth weight. The accuracy of the clinical method, with variations of $5 \%, 10 \%$ and $15 \%$ between estimated and observed weights, was $55.3 \%, 73 \%$ and $86.7 \%$, respectively. Those of the US were $60.7 \%, 75.4 \%$ and $91.1 \%$, respectively. When comparing both sets of figures, values were not different from a statistical standpoint.

Conclusion: the clinical evaluation has shown to be accurate, similarly to the US, when calculating the birth weight.

KEY WORDS: Fetal weight. Ultrasonography. Normal pregnancy.

\section{Referências}

1. ACOG. Ultrasonography in pregnancy. Technical Bulletin 187, 1983. p. 3

2. Azziz R, Smith S, Fabro S. The development and use of a standard symphysial-fundal heigh growt curve in the prediction of small for gestacional age neonates. Int $\mathbf{J}$ Obstet Gynaecol 1988, 26: 81-7.

3. Beazley JM, Underhill RA. Fallacy of fundal heigth. Br Med J 1970; 4: 404-6.

4. Belizan JM, Villar J, Nardin JC, Malamud J, Vicuna
LS. Diagnosis of intrauterine growth retardation by a simple clinical method: measurement of uterine heigth. Am J Obstet Gynecol 1978; 131:643-46.

5. Calvert JP, Crean EE, Newcombe RG, Pearson JF. Antenatal screening by measurements of symphysis-fundus heigth. Br Med J 1982; 25: 846-49.

6. Chamberlain PF. Manning FA, Morrison I, Harman CR, Lange R. Ultrasound evaluation of amniotic fluid volume. I. The relationship of marginal and decreased amniotic fluid volumes to perinatal outcome. Am J Obstet Gynecol 1984; 150:245-9.

7. Chamberlain PF, Manning FA, Morrison I, Harman CR, Lange R. II Ultrasound evaluation of amniotic fluid volume. The relationship of marginal and decreased amniotic fluid volumes to perinatal outcome. Am J Obstet Gynecol 1984; 150:250-4.

8. Chauan SP, Lutton PM, Bailey KJ, Guerrieri JP, Morrisson JC. Intrapartum clinical, sonographic, and parous patients's estimates of newborn birth weight. Obstet Gynecol 1992; 79:956-8.

9. Dare FO, Ademowore AS, Ifaturoti OO, Nganwuchu A. The value of symphysio-fundal height/ abdominal girth measurements in predicting fetal weight. Int J Obstet 1990, 31: 243-48.

10.Johnson RW, Toshach CE, Mich S. Estimation of fetal weigth using longitudinal mensuration. Am J Obstet Gynecol 1954; 68:89-6.

11.Loeffler FE: Clinical foetal weigth prediction. J Obstet Gynaecol Br Commonw 1967; 74:475-7.

12.Patterson RM. Estimation of fetal weight during labor. Obstet Gynecol 1985;65:330-2

13.Rezende J, Montenegro CAB. Obstetrícia Fundamental. 5a edição. Rio de Janeiro: Guanabara, 1987. p.121

14.Rosemberg K, Grant JM, Tweedie I, Aitchison T, Gallagher F. Measurements of fundal height as a screening test for fetal growth retardation. Br J Obstet Gynaecol 1982, 89:447-50.

15.Shepard MJ, Richards VA, Berkowitz RL. An evaluation of two equations for predicting fetal weight by ultrasound. Am J Obstet Gynecol 1982; 142:47-50.

16.Woo JSK, Ngan HYS, Au KKL, Fung KP, Wong VCW: Estimation of fetal weigth in utero from symphysis-fundal heigth and abdominal girth measurements. Aust NZ J Obstet Gynaecol 1985, 25:268-71. 